\title{
IBM SUPPLY-CHAIN NETWORK OPTIMIZATION WORKBENCH: AN INTEGRATED OPTIMIZATION AND SIMULATION TOOL FOR SUPPLY CHAIN DESIGN
}

\author{
Hongwei Ding \\ Wei Wang \\ Jin Dong \\ Minmin Qiu \\ Changrui Ren \\ IBM China Research Laboratory \\ Building 19 Zhongguancun Software Park, 8 Dongbeiwang West Road, \\ Haidian District, Beijing 100094, P. R. China
}

\begin{abstract}
The IBM Supply-chain Network Optimization Workbench (SNOW) is a software tool that can help a company make strategic business decisions about the design and operation of its supply chain network. The tool supports supply chain analysis with integrated network optimization and simulation capability. Mathematical programming models are used to first help identify some cost-effective scenarios from a large number of candidates. Optimization results are then converted to simulation models automatically for more detailed analysis with taking into account operational policies and uncertainties. The tool was applied to analyze both IBM's internal supply chains and external clients' supply chains. The combination of optimization and simulation demonstrates great value in real business cases.
\end{abstract}

\section{INTRODUCTION}

In 2002, American companies spent $\$ 910$ billion, or about $8.7 \%$ of the United States gross domestic product (GDP), on business logistics systems (Shi et al. 2004), which contained the warehousing, transportation costs, shipping related costs and logistic administration costs. In Singapore, the transportation and communication industry sector contributed about 10.8\% of the GDP in 2003 (Cheong 2005). Considering the importance and the influence of supply chain management (SCM), manufacturers and retailers like the IBM and Wal-Mart have paid great efforts to handle the flow of products efficiently and coordinate the management of supply chain smoothly.

Therefore effective and efficient supply chain management has earned lots of research attentions in the leading manufacturers, distributors, and retailers. Typically, supply chain decisions can be categorized into three sets based on the horizons of their effects (Shi et al. 2004), i.e., strategic, tactical, and operational decisions. The strategic decisions focus on the long-term effects on a company and consider the global economic environments, e.g. supply chain network configuration, strategic supplier selection, etc. The tactical level decisions include selecting specific locations among all the potential ones, searching for the optimal allocation and transportation policies in the supply chain network. The tactical decisions are made once a year or more. The operational level decisions, such as scheduling, are usually made on a daily basis to handle the detailed operations of a company.

Design of a supply chain involves determination of i) the number and location of supply chain facilities, including plants, distribution centers, warehouses and depots, ii) the transportation links and modes between facilities, and iii) the policies to operate a supply chain, such as inventory control policy, carrier loading policy etc. The first two types of decisions are often strategic decisions, while the determination of policies are more at the tactical and/or operational level. These decisions are often mixed together in the real business cases. For example, in a distribution network rationalization scenario, clients request for decision support on determining the number and location of their distribution centers (DC) across the nation. In the meantime, they need to set the inventory control policy for each DC and each SKU (stock keeping unit). An integrated analysis with optimization and simulation is necessary in this case, where optimization is used to identify a couple of most cost-effective network configurations from the huge set of candidates, and simulation is then used to evaluate performance of different policies for the networks identified.

In this paper, we present a software tool "IBM Supplychain Network Optimization Workbench" (SNOW), which supports integrated optimization and simulation analysis. IBM SNOW is a software tool that can help a company make strategic business decisions about the design and operation of its supply chain network. Mathematical pro- 
gramming models are used to first help identify some costeffective scenarios from a large number of candidates. Optimization results are then converted to simulation models automatically for more detailed analysis with taking into account operational policies and uncertainties.

In the remaining part of the paper, Section 2 gives a brief literature review in the field of network optimization, simulation and simulation-based optimization. An overview of the tool is given in Section 3. Section 4 details the simulation framework employed in the tool and highlights some novel ideas about connecting optimization and simulation. Some conclusions and perspectives are given in Section 5.

\section{REVIEW OF OPTIMIZATION, SIMULATION AND THEIR COMBINATION}

\subsection{Supply Chain Network Optimization}

The supply chain network design problem have been studied in the academia for a long time. Geoffrion and Powers (1995) analyzed the evolution of distribution system design in the past twenty years before 1995. A number of elements are identified which have significantly contributed to the evolution of distribution systems, including the logistics functionalities, information systems, developed algorithms and enterprise management systems. They also claimed that customer service and client requests will remain as the most fundamental aspects for research.

Schmitdt (2000) present a state-of-the-art on various modeling and optimization approaches used for supply chain decision-making at strategic, tactic and operational levels. In addition to a review on existing models, the authors pointed out that decisions of different levels interact with each other. For example, strategic decisions usually impose structural constraints on low level decisions. As feedback, low level decisions and corresponding operational performances should also be taken into account by high level decision-making.

For supply chain optimization practitioners, one major obstacle is related to supply chain uncertainties and dynamics. The stochastic nature of supply chains makes most analytical models either over simplistic or computationally intractable.

\subsection{Supply Chain Simulation.}

As pointed out by Bagchi et al. (1998): "We have found that a typical strategic analysis requires a combination of optimization and simulation. Optimization technology such as linear or mixed integer programming is useful for solving well-defined mathematical problems such as warehouse location and inventory optimization. But these problems are rigidly defined and usually have simplifying assumptions in their formulations. Such problems must be solved independently and thus in a complex supply chain one must decompose all of the issues into a hierarchical sequence of problems. At best, a hierarchical approach is awkward and introduces a second level of simplifying assumptions. At worst, this approach may not be feasible if some of the problems are nonlinear or stochastic.", optimization is hardly used independently in industries. Comparing to optimization techniques, simulation is more suitable for the evaluation of complex industrial systems given a limited number of candidate scenarios.

Supply Chain Analyzer (SCA) is a comprehensive tool for supply chain simulation. SCA is also developed by IBM Research, originally for IBM internal use. SCA deploys a mix of simulation and optimization functions to model and analyze supply chain issues such as site location, replenishment policies, manufacturing policies, transportation policies, stocking levels, lead times, and customer service (Bagchi et al. 1998). A simulation tool "Supply Solver" is presented in (Schunk and Plott 2000). The tool is connected with external modules and provides optimal solution on the production and distribution allocation problem. Supply Chain Guru, developed by Promodel Corporation, is a commercial simulation tool for supply chain design and planning, with a specific module for inventory planning (Supply Chain Guru 2006).

Besides those simulation software, there are a number of supply chain simulation framework/library have been developed. Swaminathan (1998) describe a supply chain modeling framework in which supply chain models are composed from software components that represent types of supply chain agents, their constituent control elements, and their interaction protocols. Rossetti (2003) discusses the design, development and testing of a prototype objectoriented framework for per-forming supply chain simulation. Rossetti (2006) discusses the design and use of an object-oriented framework for simulating multi-echelon inventory systems.

\subsection{Simulation-based Optimization}

The conception of combining simulation and optimization is not new. A general simulation-based optimization method consists of an optimizer guiding the search direction and a simulation module used performance evaluation. Compared with mathematical programming techniques, simulation-based optimization methods replace the analytical objective function and constraints by one or more simulation models. The decision variables are the conditions under which the simulation is run. Iteratively, the output of the simulation is used by the optimizer to provide feedback on the progress of the search for an optimal solution. Nevertheless, the optimality of the solution found by a simulation-based optimization method is often not guaranteed. 
Many different methods have been developed in the literature for guiding the search direction in simulationbased optimization. These methods include gradient-based approaches, response surface methodology, sample path optimization, etc (Fu 2002). These methods vary essentially in their optimization strategies and each has its advantages and disadvantages. For example, infinitesimal perturbation analysis (IPA), a gradient based search method which estimates all gradients of the performance function in a single simulation run, possesses an advantage of computational efficiency. However, the estimators derived using IPA are often biased and inconsistent (Ho and Cao 1991).

According to an empirical comparison (Lacksonen 2001) of four search algorithms (pattern search, simplex, simulated annealing, genetic algorithm) used for simulation-based optimization, genetic algorithms showed its capability to robustly solve large problems with non-numeric variables, and performed well over the others in solving a variety of problems. A genetic algorithm based multiobjective optimization method has been applied to facilitate decision-making on supplier selection problems (Ding et al. 2004). Paul and Chanev (1998) developed a simple real-coded genetic algorithm to optimizer the control parameters of a steelworks simulation model.

\section{OVERVIEW OF THE IBM SUPPLY-CHAIN NETWORK OPTIMIZATION WORKBENCH}

\subsection{Tool Architecture}

The IBM Supply-chain Network Optimization Workbench is a software tool which supports integrated optimization and simulation analysis. Accordingly, there are two key models, respectively an optimization model and a simulation model. As shown in the software architecture overview (Figure 1), the two models are connected with each other directly. Actually, the approach used in IBM SNOW is different from the simulation-based optimization methods, in which the two models are usually tightly bundled. More specifically, optimization and simulation are run in a sequential way, i.e. firstly a network optimization will be conducted, then the optimization result will be converted to a simulation model automatically for evaluation. More detailed description of the connection between optimization model and simulation model is given in the following section.

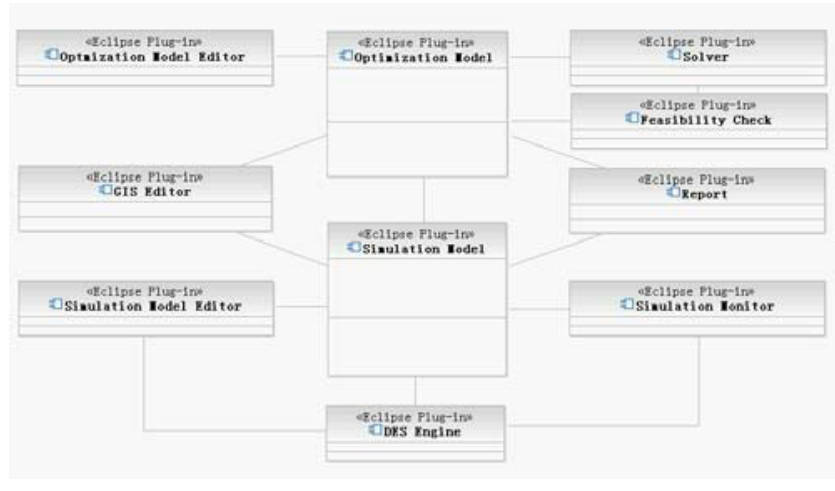

Figure 1: Tool architecture overview

In addition to the two models, the SNOW tool provides a number of graphic user interfaces to facilitate data input, network representation and parameter setting. Supply chain data could be input manually using the tool interface, or automatically imported from Excel files or Access database. Moreover, a Geographic Information System (GIS) is integrated for graphical representation, which enables a flexible and precise representation of the actual supply chain networks. As long as the longitude and latitude of each facility is set, the whole network can be displayed in the map. Another advantage of using GIS for network display is that users could zoom into very local region seamlessly. Figure 2 illustrates a supply chain network modeled in the SNOW tool and displayed in the GIS editor.

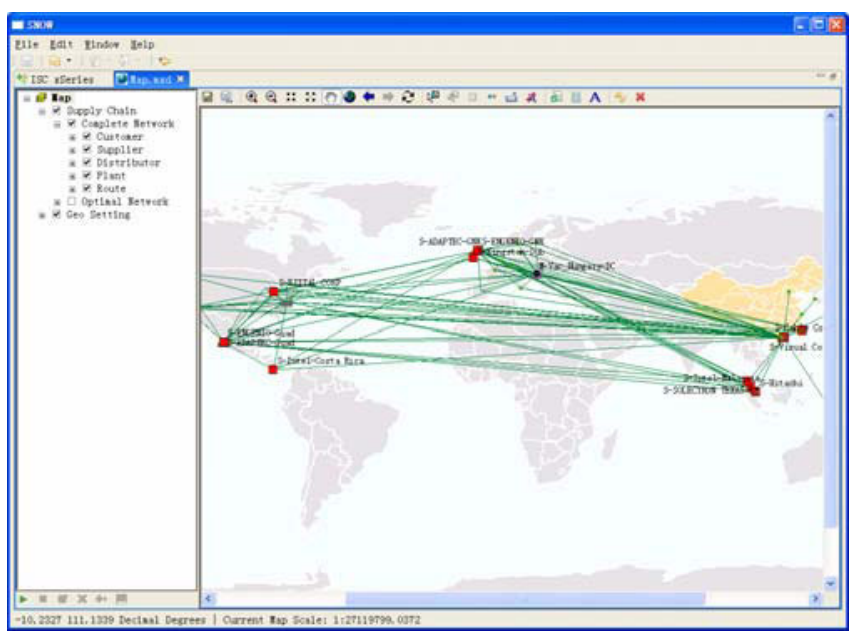

Figure 2: Network representation in the geographic information system

\subsection{Optimization Model}

A variety of mathematical models and methods are built in the SNOW tool. The key enabling component is a mixed integer programming model addressing multi-commodity facility location and network flow optimization problem. 
Due to confidentiality, no detailed mathematical formulation is provided in this paper. However, we focus on introducing the supply chain design problem addressed by the tool. The objective is to minimize the total supply chain cost, with respect to a variety of constraints. More specifically, the total cost function is composed of procurement cost, transportation cost, assembly cost, operation cost, facility fixed cost, facility investment cost, inventory carrying cost and tax. A large number of constraints have been taken into account in the model, including supply capacity, assembly capacity, transportation capacity, single sourcing rule, etc. Figure 3 shows a screenshot of the data input interface for the optimization model.

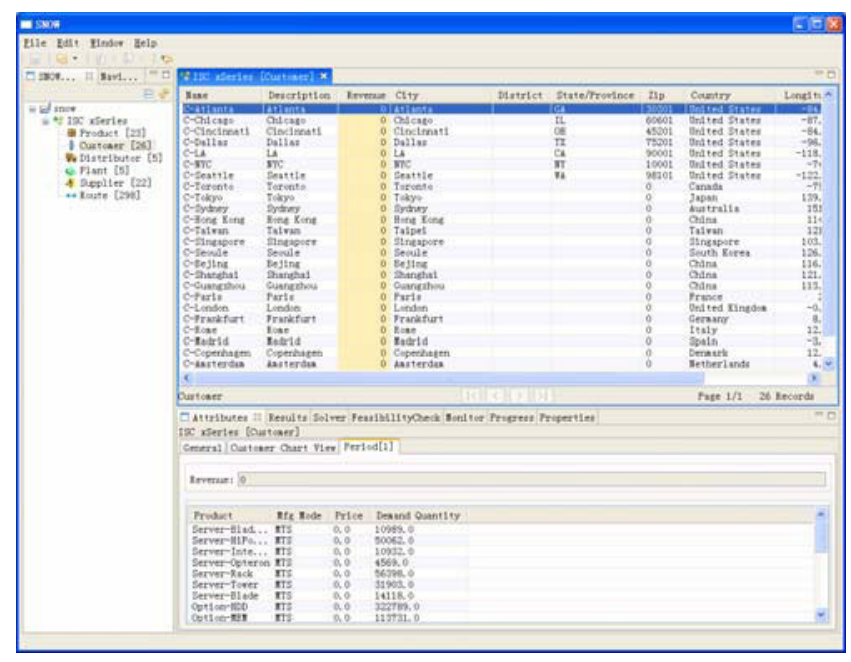

Figure 3: Snapshot of the optimization data input interface

A couple of algorithms are developed and integrated in the tool for problem solving, including mixed integer programming, linear programming, heuristics and metaheuristics. Two solvers are integrated in the tool for small and medium scale problem solving. One solver is a commercial solver, called ILOG Cplex. The other is an open source solve, called Lp_Solve. In addition to the two builtin generic solvers, we have also developed some hybrid algorithms for large-scale network optimization.

\subsection{Implementation}

The tool is implemented using Eclipse, an open-source platform for Java application development. More specifically, the tool is developed based on several basis provided by Eclipse Foundation, including Eclipse Platform (Eclipse 2007), Eclipse Modeling Framework (EMF 2007) and Graphical Editing Framework (GEF 2007).

Eclipse is an open source community whose projects are focused on building an open development platform comprised of extensible frameworks, tools and runtimes for building, deploying and managing software across the lifecycle (Eclipse 2007). Eclipse Modeling Framework
(EMF) is a modeling framework and code generation facility for building tools and other applications based on a structured data model. Graphical Editing Framework (GEF) allows developers to create a rich graphical editor from an existing application model.

The whole optimization and simulation model is built upon EMF. User interfaces of the tool are created with GEF. Since the optimization and simulation model are quite complex, we built it with UML2. The power of EMF is that UML2 models can be directly translated to EMF model, and then the Java source code can be generated accordingly.

\section{COMPONENT-BASED SIMULATION}

\subsection{Simulation Model Overview}

The simulation model employed in the SNOW tool is a component-based simulation model. Figure 4 depicts the architecture of the simulation model. There is a generic discrete event simulation (DES) engine for event scheduling and time processing. A simulation monitor is also developed to monitor the simulation process and key performance indicators (KPI). Simulation report is generated after simulation runs with statistics information on KPIs like supply chain cost, order fill-rate, stock out rate, etc.

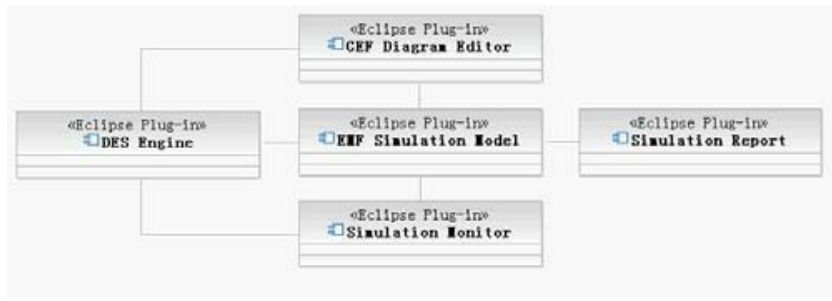

Figure 4: Architecture of the Simulation Model

One differentiating feature of the simulation model in SNOW is that the model is a multi-period model. This feature comes from the optimization model, because network optimization problems are usually multi-period in order to take into account the seasonality of demand and supply. Accordingly, the whole simulation horizon is split into multiple period. For each period, users could have a set of parameters, such as inventory control policy and parameters, transportation lead-time.

\subsection{Supply Chain Facilities}

Four types of supply chain facilities are defined in the tool, respectively: Customer, Supplier, Distribution Center (DC), and Plant. The class diagram is shown in Figure 5. 


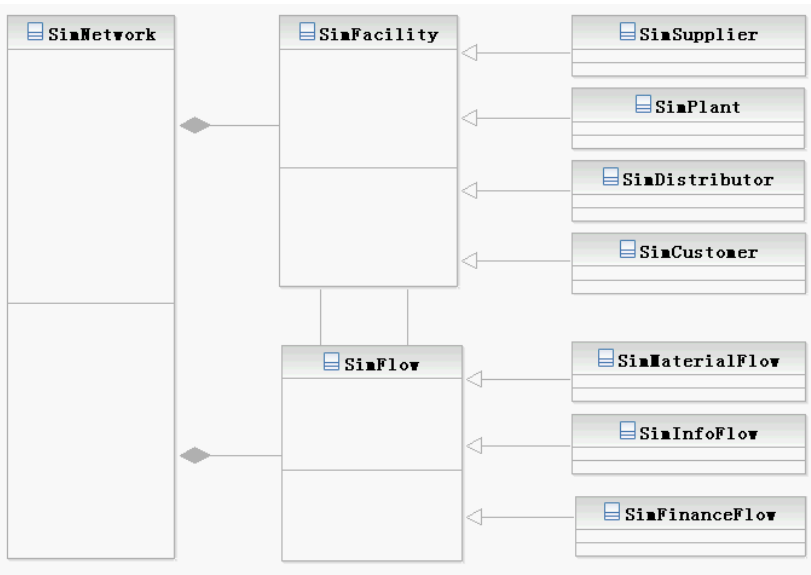

Figure 5: Class diagram of supply chain facilities

In the era of supply chain competition, decision makers usually would like to analyze the performance of a specific enterprise in a supply chain which is composed of a group of companies. Hence all supply chain facilities can be classified into two categories: external and internal. Customer and Supplier are used to model external facilities. More specifically, they represent the most down-flow and up-flow facilities in the supply chain respectively. DC and Plant are used to model internal facilities. The difference between them is that Plant can transform products but DC can not.

More specifically, Customer represents the external demand source. It can be end user, retailer, or wholesaler. It can even be distribution center or plant of external company. Supplier represents the external supplier, which has no up-flow suppliers. But Supplier doesn't always have unlimited inventory, supply capacity can be specified. DC represents internal warehouse, retailer or wholesaler. Products are shipped into DC from up-flow facilities, and then shipped out to down-flow facilities to fulfill their demands. Plant could be regarded as a more comprehensive DC, with the production capability.

\subsection{Operation Components}

As we all know, to simulate a supply chain, it requires not only the modeling of supply chain facilities, but also the modeling of operational policies in order to drive order flow, material flow and financial flow. Six types of components are defined in SNOW, including Supply component, Sourcing component, Inventory component, Transportation component, Manufacturing component, and Finance component. Supply component and Transportation component deal with down-flow facilities. Sourcing component deal with up-flow facilities. Inventory component and Manufacturing component deal with issues inside the owner facility. Finance component deals with inside and outside finance related issues.
In terms of functionality of each component, Supply component handles incoming customer orders. Sourcing component generates customer orders and replenishment orders. Transportation component handles transportation tasks. It receives transportation requests, and then manages to ship products to down-flow facilities according to requests. Inventory component holds stock of products, which includes raw materials and finished goods. Manufacturing component provides the capability of transform products from raw materials to finished goods. Finance component handles the cash flow between different facilities, and also different enterprises in a supply chain.

\subsection{Connection between Optimization Model to Simulation Model}

The connection between optimization model and simulation model has been a challenge during the research and development of the tool. We are still developing and integrating more flexible means to enhance the connection. In this paper, we introduce the mechanism that has been built in the tool and verified in a couple of customer projects.

The essential idea behind is that once the optimization result is produced by the optimizer, it is converted to a simulation model automatically. After the automatic conversion is conducted, the additional simulation-specific attributes are specified according to some rules, which are also pre-set by end user. After the simulation modeling phase, we conduct the simulation, get the simulation results, and compare them with the optimization results in order to validate the best solution. These steps are further elaborated as follows.

The first step is to convert network structure. The optimization model and simulation model both define facility networks. These two networks are very similar, both consists of four kinds of facilities: Supplier, Plant, Distributor, and Customer. Hence the network structure can be transformed directly. What's more, the facilities suggested to be shutdown in optimization results will not be mapped to simulation model, and the facilities suggested to be started will be.

The second step is to convert customer demands. In the optimization model, aggregated demand quantity are defined for each period and for each product. While in simulation model, for generating demand event, we need to know the quantity for each product in customer order, as well as the interval between orders. So we split each period into several segments, and the aggregate demand quantity are assigned to these segments with respect to some predefined rules. For each segment, one customer order will be generated.

The third step is to convert supplier selection policies. Customer orders will be sent to up-flow facilities. In the logistics network, each customer has several up-flow facilities, which can be distributors or plants. So supplier selec- 
tions must be in place for routing these orders. The default supplier selection policy will forward orders to all up-flow facilities, but the quantity rates of orders are different. The optimization results contain information about the transportation quantity on each route, which are used to determine the related procurement quantity.

The fourth step is to convert product-period related attributes. There is a global calendar in the optimization model. It assumes all periods have the same length, and all periods with same period index have the same start date and end date. Although this assumption can cover most cases, we are seeking more flexibilities in the simulation model. So all periods in the simulation model are independent with each other and their start time and end time can be set freely. What's more, in the optimization model, it is a facility-period-product structure, which means first we define the periods for each facility, and then within each period, we define the attributes associated with each product. But in the simulation model, it is a facilityproduct-period structure, so for each product, we can define multiple periods, and then define attributes associated with the periods.

The fifth step is to define additional attributes. The simulation model needs much more attributes than optimization model. So we need to define some default values for them, such as default intervals to check customer order queues, default inventory control policies, etc. The model with these default values can be simulated directly, but it's expected for the analyst to modify them. Figure 6 demonstrates the interface of the simulation model.

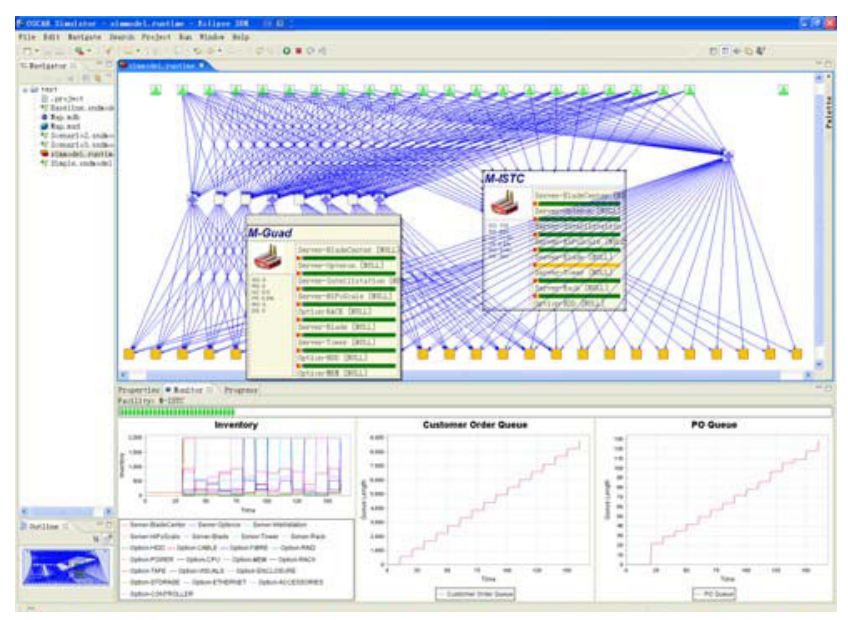

Figure 6: Network simulation model converted from optimization results

\section{CONCLUSIONS AND PERSPECTIVES}

This paper gives an overview of the software tool IBM Supply-chain Network Optimization Workbench (SNOW), developed by IBM Research. The IBM SNOW supports an integrated optimization and simulation analysis of supply chains. It has been used for the improvement of IBM's internal supply chains and also for the optimization of outside clients' supply chains. Practice demonstrate that the combination of simulation and optimization could help decision makers gain much insights from a real supply chain and finally improve its efficiency and effectiveness.

\section{REFERENCES}

Bagchi S., S. J. Buckley, M. Ettl, and G. Lin. 1998. Experience using the IBM Supply Chain Simulator. Proceedings of the 1998 Winter Simulation Conference, 1387-1394.

Cheong Lee Fong. 2005. New Models in Logistics Network Design and Implications for 3PL Companies. Ph.D. Dissertation of SINGAPORE-MIT ALLIANCE.

Ding H., L. Benyoucef and X. Xie. 2004. A multiobjective optimization method for strategic sourcing and inventory replenishment. Proc. of 2004 IEEE International Conference on Robotics and Automation, 2711-2716, New Orleans, U.S.A.

Eclipse 2007. Available via <http://www. eclipse. org > [accessed March 10, 2007].

EMF 2007. Available via <http://www.eclipse. org/emf> [accessed March 10, 2007].Fu M. C.. 2002. Optimization for simulation: Theory vs. Practice. INFORMS Journal on Computing 14(3): 192-215.

GEF 2007. Available via <http://www.eclipse. org/gef> [accessed March 10, 2007].

Geoffrion A. M. and R. F. Powers. 1995. Twenty years of strategic distribution system design: An evolutionary perspective. Interfaces 25:105-128.

Ho, Y. C. and X. R. Cao. 1991. Perturbation analysis of discrete event dynamic systems. Kluwers Academic Publishers.

Lacksonen T. 2001. Empirical comparison of search algorithms for discrete event simulation. Computers \& Industrial Engineering. 40:133-148.

Manuel D. Rossetti, Mehmet Miman, Vijith Varghese, and Yisha Xiang. 2006. An object-oriented framework for simulating multi-echelon inventory systems. Winter Simulation Conference 2006: 1452-1461.

Paul R. J. and T. S. Chanev. 1998. Simulation optimization using a genetic algorithm. Simulation Practice and Theory, 6: 601-611.

Rossetti, M. D. and Chan H.T. 2003. A Prototype ObjectOriented Supply Chain Simulation Framework. Proceedings of the 2003 Winter Simulation Conference, 1612-1620.

Schunk D. and B. Plott. 2000. Using Simulation to Analyze Supply Chains. Proceedings of the 2000 Winter Simulation Conference, 1095-1100.

Shi L., R. R. Meyer, M. Bozbay, and A. J. Miller. 2004. A nested partitions framework for solving large-scale multicommodity facility location problem. Journal of 
Systems Science and Systems Engineering 13(2): 158179.

Supply Chain Guru. 2006. Available via $<$ http:// www.llamasoft.com/guru.html> [accessed May 16, 2006].

Swaminathan, J. M., Smith, S. F. and Sadeh, N. M. 1998. Modeling Supply Chain Dynamics: A Multiagent Approach. Decision Science 29(3): 607-632.

\section{AUTHOR BIOGRAPHIES}

HONGWEI DING is a Researcher at IBM China Research Laboratory. He received his Ph.D. in Automation from INRIA (French National Institute of Computer Science \& Control), France. Before joined IBM, he was a researcher at INRIA. His research interests include supply chain modeling, optimization and simulation. His e-mail address is <dinghw@en.ibm.com>.

WEI WANG is a Researcher at IBM China Research Laboratory. He joined IBM Research in 2005 after receiving his master degree in Control Science and Engineering from Tsinghua University in Beijing, P. R. China. Currently, his research interests include supply chain simulation and optimization, performance management, and business process management. His e-mail address is <wangwcrl@cn.ibm.com>.

JIN DONG, Manager of Supply Chain Management and Logistics Research in IBM China Research Laboratory. He received his Ph.D. degree in Tsinghua University from P.R. China in 2001. Before joined IBM, he was the Research Assistant Professor in Industrial Engineering Department of Arizona State University in USA. His e-mail address is <dongjin@cn.ibm.com>.

MINMIN QIU is a Researcher at IBM China Research Laboratory. He joined IBM Research in 2006 after receiving his Ph.D. degree in Industrial Engineering from Tsinghua University in Beijing, P.R. China. His research interests include inventory optimization, machine learning, supply chain optimization and simulation. His e-mail address is<qiumm@cn.ibm.com>.

CHANGRUI REN is a Researcher at IBM China Research Laboratory. He joined IBM Research in 2005 after receiving his Ph.D. degree in Control Science and Engineering from Tsinghua University in Beijing, P. R. China. His research interests include supply chain management, logistics network design, performance management, and business process management. He is currently working on an end-to-end supply chain transformation methodology and tool. His e-mail address is <rencr@cn.ibm.com>. 